\title{
Abbreviations Used in This Manual
}

APPENDIX 1A

A adenine or adenosine

Ac acetyl

ADA adenosine deaminase

ADP adenosine 5'-diphosphate

AFM atomic force microscopy

AMP adenosine 5'-monophosphate

3-APA 3-aminopicolinic acid

Ar aryl

ATP adenosine 5'-triphosphate

ATPase adenosine triphosphatase

BCIP 5-bromo-4-chloro-3-indolyl

phosphate

BMPM 1,1-bis(4-methoxyphenyl)-1-

pyrenylmethyl

BSA bovine serum albumin

Bu butyl

Bz benzoyl

C cytosine or cytidine

CD circular dichroism

cDNA complementary deoxyribonucleic

acid

CDP cytidine 5'-diphosphate

$C^{\mathbf{e x}}{ }_{\mathbf{p}}$ excess heat capacity

Ci curie

CID collision-induced dissociation

CMCT 1-cyclohexyl-3-(2-morpholinoethyl)carbodiimide metho- $p$-toluene sulfonate

CMP cytidine 5'-monophosphate

COSY correlated spectroscopy

CPG controlled-pore glass

CPK Corey-Pauling-Koltun (molecular

models)

cpm counts per minute

CPMB Current Protocols in Molecular

Biology

CSP calf spleen phosphodiesterase

Ctmp 1-(2-chloro-4-methylphenyl)-4-

methoxypiperidin-4-yl

CTP cytidine 5'-triphosphate

dA deoxyadenosine

dAMP deoxyadenosine monophosphate

dATP deoxyadenosine triphosphate

DBMB 2-(dibromomethyl)benzoyl

DBU 1,8-diazabicyclo[3.4.0] undecene-

7-ene

dC deoxycytosine

DCA dichloroacetic acid

DCM dichloromethane
dCMP deoxycytidine monophosphate

dCTP deoxycytidine triphosphate

DDQ 2,3-dichloro-5,6-dicyano-1,4-benzo

quinone

DEAD diethyl azodicarboxylate

DEC 1-(3-dimethylaminopropyl)-3-

ethylcarbodiimide hydrochloride

DEPC diethylpyrocarbonate

dG deoxyguanosine

DG distance geometry

dGMP deoxyguanosine 5'-monophosphate

dGTP deoxyguanosine 5 -triphosphate

DIP 4,7-diphenyl-1,10-phenanthroline

DMAEDE 2 - $O-2$-[2-( $N, N$-dimethylamino)

ethyloxy] ethyl

DMAoE 2'-O-[2-( $N, N$-dimethylami-

nooxy)ethyl

DMAP 4-dimethylaminopyridine

DMBOC 3',5'-dimethoxybenzoinoxycar-

bonyl

DMF dimethylformamide

DMS dimethyl sulfate

DMSO dimethylsulfoxide

DMTr 4,4'-dimethoxytrityl (also DMT)

DNA deoxyribonucleic acid

DNBSB 2-(2,4-dinitrophenylsulfenyloxy-

methyl)benzoyl

DNPEOC 2-(2,4-dinitrophenyl)ethoxycar-

bonyl

dNTP deoxynucleoside triphosphate

dpm disintegrations per minute

DSC differential scanning calorimetry

dsDNA double-stranded DNA

DTMT 2-(iso-

propylthiomethoxymethyl)benzoyl

Dtr 4-decyloxytrityl

DTT dithiothreitol

dTTP deoxythymidine triphosphate

dUTP deoxyuridine triphosphate

EDTA ethylenediaminetetraacetic acid

ESI electrospray ionization (mass

spectrometry)

Et ethyl

EtBr ethidium bromide

Et3N triethylamine

$\mathbf{E t}_{2} \mathbf{O}$ ethyl ether

EtOAc ethyl acetate

EtOH ethanol

FAM 5-carboxyfluorescein
Abbreviations and Useful Data

Current Protocols in Nucleic Acid Chemistry (2000) A.1A.1-A.1A.3

Copyright $(\odot 2000$ by John Wiley \& Sons, Inc. 
FMOC 9-fluorenylmethoxycarbonyl

Fpmp 1-(2-fluorophenyl)-4-methoxypiperidin-4-yl

FTICR Fourier-transform ion-cyclotron

resonance mass spectrometry

G guanine or guanosine

GDP guanosine $5^{\prime}$-diphosphate

GMP guanosine 5 '-monophosphate

GTP guanosine $5^{\prime}$-triphosphate

HEG hexa(ethylene glycol)

HEPES $N$-2-hydroxyethylpiperazine- $N N^{\prime}-2$ ethanesulfonic acid

HEPPSO $N$-(2-hydroxyethyl)piperazine$N^{\prime}$-(2-hydroxypropanesulfonic acid)

3-HPA 3-hydroxypicolinic acid

HPLC high-performance liquid chromatography

HQDA hydroquinone- $O, O^{\prime}$-diacetic acid

HSDIS hydroxystyryldiisopropylsilyl

HSDMS hydroxystyryldimethylsilyl

$\boldsymbol{i}$-Bu isobutyl

$\boldsymbol{i}$-Pr isopropyl

ITC isothermal titration calorimetry

IUB International Union of Biochemistry

IUPAC International Union of Pure and Applied Chemistry

JOE 6-carboxy-4', 5'-dichloro-2' , $^{\prime}$-dimethoxyfluorescein

LCAA long-chain alkylamine

LNA locked nucleic acid

L-TOF linear time-of-flight (mass spectrometry)

MALDI matrix-assisted laser desorp-

tion/ionization (mass spectrometry)

MDMP 1,5-dimethoxylcarbonyl-3-

methoxypentan-3-yl

Me methyl

MeNPOC methylnitropiperonyloxycar-

bonyl

MeOH methanol

Me-P methylphosphonate

MMI methylenemethylimino

MMTr 4-monomethoxytrityl

MOPS 3-( $N$-morpholino)propane sulfonic

acid

MOTr 4-methoxy-4'-octyloxytrityl

MOX 9-(p-anisyl)xanthen-9-yl

MS mass spectrometry

MSNT 1-(mesitylene-2-sulfonyl)-3-nitro-

1,2,4-triazole

MTHP 4-methoxytetrahydropyran-4-yl

MTMB 2-(methylthiomethoxymethyl)-

butyryl

MTMT 2-(methylthiomethoxymethyl)

benzoyl

MWCO molecular weight cutoff
NAB nucleic acid builder (computer program)

NBT nitroblue tetrazolium

n-Bu n-butyl

NDB Nucleic Acid Database

NMR nuclear magnetic resonance

NOE nuclear Overhauser effect

NOESY nuclear Overhauser effect spec-

troscopy

N-P phosphoramidate

NPE 4-nitrophenylethyl

NPEOC 2-(4-nitrophenyl)ethoxycarbonyl

NPOC nitrophenyloxycarbonyl

nt nucleotide

PA picolinic acid

PAGE polyacrylamide gel electrophoresis

PAPOC $p$-phenylazophenyloxycarbonyl

PASS product-anchored sequential

synthesis

PBS phosphate-buffered saline

PCR polymerase chain reaction

PFPC bis(pentafluorophenyl) carbonate

Ph phenyl

PIPES piperazine- $N, N^{\prime}$-bis (2-ethanesul-

fonic acid)

pixyl 9-phenylxanthen-9-yl

PMSF phenylmethylsulfonylfluoride

PNA peptide nucleic acid

PNK polynucleotide kinase

Pr propyl

PS-PTFE polystyrene-polytetrafluoro-

ethylene

PTMT 2-(isopropylthiomethoxymethyl)benzoyl

Pu purine

Px 9-phenylxanthen-9-yl

Py pyrimidine

RFP radiofrequency plasma

rMD restrained molecular dynamics

RNA ribonucleic acid

RNAP RNA polymerase

$\mathbf{R}, \mathbf{R}^{\prime}$ alkyl

RT-PCR reverse transcription/polymerase

chain reaction

SA sinapinic acid

SDS sodium dodecyl sulfate

sec-Bu sec-butyl

SELEX Systematic Evolution of Ligands

by Exponential Enrichment

3SR self-sustained sequence replication

SSC sodium chloride/sodium citrate (buffer)

ssDNA single-stranded DNA

SSPE saline sodium phosphate/EDTA

buffer

STM scanning tunneling microscopy

SVPD snake venom phosphodiesterase

TAE Tris/acetate/EDTA (buffer) 
TBAF tetrabutylammonium fluoride

TBDMS tert-butylmethylsilyl

TBE Tris/borate EDTA (buffer)

TBF 4-[17-tetrabenzo $(a, c, g, i)$ fluorenylmethyl]

TBS Tris-buffered saline

$\boldsymbol{t}$-Bu tert-butyl

TCA trichloroacetic acid

TCEP tris-(2-carboxyethyl)phosphine

TE Tris/EDTA (buffer)

TEA triethylamine

TEMED $N, N, N^{\prime}, N^{\prime}$-tetramethylethylene-

diamine

TES $N$-tris(hydroxymethyl)methyl-2-aminoethanesulfonic acid

TFC triplex-forming circle

THF tetrahydrofuran-2-yl
THP tetrahydropyran-2-yl

TLC thin-layer chromatography

TMP 3,4,7,8-tetramethyl-1,10-phenanthroline, thymidine $5^{\prime}$-monophosphate

TMSE trimethylsilylethyl

TMTR trimethoxytrityl

TOF time-of-flight (mass spectrometry)

$\boldsymbol{t}$-Pac tertiary butylphenoxyacetyl

Tris tris(hydroxymethyl)aminomethane trityl diphenylmethyl

TsOH toluene-4-sulfonic acid

TTP thymidine $5^{\prime}$-triphosphate

$\mathbf{U}$ uracil

UDP uridine 5 -diphosphate

UMP uridine $5^{\prime}$-monophosphate

UTP uridine 5 'triphosphate

UV ultraviolet 